\title{
MÉTODOS DE SECAGEM DE AMOSTRAS DE FOLHAS DE CAFÉ, MANGA E AMORA NA DETERMINAÇÃO DA MATÉRIA SECA E NOS TEORES DE MACRONUTRIENTES
}

\author{
DIAS, José Luiz Carvalho de Souza ${ }^{1}$ \\ PRADO, Renato de Mello ${ }^{2}$ \\ ROCHA, Lucian Cima de Melo ${ }^{3}$ \\ VIEIRA, Douglas Silva Tobias ${ }^{4}$
}

\begin{abstract}
RESUMO: A secagem de amostras foliares com emprego do forno de microondas pode ser alternativa viável para obtenção rápida dos resultados da análise foliar. Objetivou-se comparar o processo de secagem em FMO e em estufa de ventilação de ar forçada na determinação da massa seca e nos teores de macronutrientes foliares das culturas de café, manga e amora. Os tratamentos constituíram-se por dois métodos de secagem, estufa de circulação de ar forçada regulada a uma temperatura de $70^{\circ} \mathrm{C}$ e o FMO, onde cada amostra foi submetida à secagem a 3 ciclos de 5 minutos, 1 ciclo de 3 minutos, 1 ciclo de 2 minutos e 1 ciclo de 1 minuto, atingindo massa constante. $\mathrm{O}$ delineamento experimental utilizado foi um arranjo hierárquico $2 \times 3$ ( 2 métodos de secagem e 3 espécies), com 6 repetições. O uso de FMO e da estufa com circulação de ar forçada na determinação da massa seca e nos teores de macronutrientes de folhas das culturas de café, manga e amora foram semelhantes, indicando que o uso do FMO é uma alternativa viável para uso em laboratórios de análise foliar.
\end{abstract}

Palavras-chave: Métodos de secagem. Matéria seca. Análise química.

\section{METHODS OF DRYING LEAF SAMPLES OF COFFEE, MANGO AND BLACKBERRY IN THE DETERMINATION OF DRY MATTER AND MACRONUTRIENTS CONTENTS}

\begin{abstract}
SUMMARY: The drying of leaf samples with use of microwave oven can be a viable alternative for obtaining rapid results of leaf analysis. The objective was to compare the drying process in FMO and greenhouse forced air ventilation in the determination of dry matter and macronutrient leaf crops of coffee, mango and blackberry. The treatments consisted of two drying methods, greenhouse circulation of air regulated to a temperature of $70^{\circ} \mathrm{C}$ and FMO, where each sample was subjected to drying to 3 cycles of 5 minutes, 1 cycle of 3 minutes, 1 cycle 2 minutes and 1 cycle of 1 minute, reaching constant weight. The experiment was a hierarchical arrangement $2 \times 3$ (two drying methods and three species), with six replicates. The use of FMO and greenhouse with forced air circulation in the determination of dry weight and macronutrient content of leaves of the crops of coffee, mango and blackberry were similar, indicating that the use of FMO is a viable alternative for use in laboratories leaf analysis.
\end{abstract}

Keywords: Drying methods. Dry matter. Chemical analysis.

\footnotetext{
${ }^{1}$ Graduando em Agronomia, Universidade Estadual Paulista, Júlio de Mesquita Filho, Faculdade de Ciências Agrárias e Veterinárias de Jaboticabal, Departamento de solos e adubos. Via de acesso professor Donato Castellane, s/n. CEP: 14884-900, Jaboticabal-SP, Brasil. e-mail: joseluizdias@ymail.com

2 Universidade Estadual Paulista, Júlio de Mesquita Filho, Faculdade de Ciências Agrárias e Veterinárias de Jaboticabal, Departamento de solos e adubos. Via de acesso professor Donato Castellane, s/n. CEP: 14884-900, Jaboticabal-SP, Brasil.. e-mail: rmprado@pq.cnpq.br

${ }_{3}^{3}$ Engenheiro Agrícola, Universidade Federal de Lavras, e-mail:dougtobias11@ @otmail.com
} 


\section{INTRODUÇÃO}

A análise química foliar é a principal ferramenta para a avaliação e manejo do estado nutricional das plantas, uma vez que a folha é o órgão que melhor reflete o estado nutricional na maioria das culturas (MALAVOLTA, 2006), isto é, respondem mais as variações no suprimento de nutrientes (GRASSI FILHO, 2008), por se tratar do centro das atividades fisiológicas dentro das plantas.

A determinação de matéria seca de espécies como café, manga e amora é realizada comumente pelo método convencional, que é o da estufa de ventilação de ar forçada, porém este método é lento, em geral 72 horas, e mudanças bioquímicas podem ocorrer durante esse período, por isso que é importante a avaliação de outros métodos de secagem. Marur E Sodek (1995) já relatavam que a secagem de material vegetal pode ser feita em forno de microondas (FMO), procedimento muito mais rápido.

É de grande importância testar métodos alternativos de secagem de amostras foliares, como o de FMO, pois com um menor tempo de secagem da amostra (entorno de 22 minutos e sem influenciar nos teores foliares de nutrientes) permitiria um preparo mais rápido da amostra, assim como resultado da análise foliar e com reflexos no diagnóstico da possível desordem nutricional da planta, possibilitando a realização de ajustes rápidos na adubação minimizando eventuais perdas na produção. Determinadas partes dos vegetais contém alto teor de amido e proteína, sendo facilmente infestadas por microorganismos e insetos, o que reduz seu valor e durabilidade (LACERDA et al. 2009). Esses materiais, quando secados em FMO, apresentam melhor conservação da cor verde e maior durabilidade do que quando secadas em estufa (CHANG et al., 1994).

Apesar de alguns trabalhos considerarem como satisfatória a determinação da MS utilizando o método convencional (NARASIMHALU et al., 1982; HIGGINS; SPOONER, 1986), durante esse processo, pode ocorrer volatilização de ácidos orgânicos e amônio (NARASIMHALU et al., 1982) favorecendo mudanças bioquímicas na composição do material (PASTORINI et al., 2002).

Apesar de todas essas vantagens, ainda são escassos os trabalhos sobre secagem em FMO e seus efeitos na qualidade do material vegetal, bem como determinação da matéria seca e nos teores de macronutrientes, principalmente em culturas como café, manga e amora.

Com este trabalho objetivou-se comparar o processo de secagem em FMO e em estufa de ventilação de ar forçada na determinação da massa seca e nos teores de macronutrientes foliares das culturas de café, manga e amora. 


\section{MATERIAL E MÉTODOS}

Foi desenvolvido um experimento em condições de laboratório da FCAV/UNESP, utilizando amostras de tecido vegetal das culturas de café, manga e amora, secas por dois métodos, convencional em estufa de circulação forçada de ar com capacidade de 68L, e o forno de microondas (FMO), contendo as seguintes características: carga máxima: $5 \mathrm{~kg}$, capacidade: 35L, tensão de alimentação: $220 \mathrm{~V}$, corrente: 13 A, freqüência: $60 \mathrm{~Hz}$ (rede), potência útil: 900W (máxima), freqüência: $2450 \mathrm{MHz}$ (operação), dimensões externas (largura: 306, profundidade: 555 e altura: 455).

Para isto, foram feitas amostragem foliar nas culturas, onde foram consideradas as indicações feitas na literatura sendo para o café coletar o 3 par a partir do ápice do ramo frutífero, da altura média da planta, no início do verão, sendo duas folhas por planta em 50 plantas por talhão, para a manga coletar folhas do meio do último fluxo de vegetação de ramos com flores na extremidade, amostrando 4 folhas por árvore, 20 plantas por talhão, e para a amora coletar folhas jovens totalmente expandidas de ramos frutíferos. Em cada cultura, foram coletadas 12 amostras com cerca de 30 folhas, portanto obtivemos 36 amostras para as três culturas. No laboratório, o material foi descontaminado pela lavagem tendo a seguinte sequência, a água corrente "destilada"; solução detergente $(0,1 \%)$; solução ácido clorídrico (0,3\%); água deionizada, conforme Prado (2008). Em seguida, as amostras foram deixadas em repouso para escorrer o excesso de água e determinado a massa fresca de cada amostra. Após isso foram aplicados os tratamentos em cada amostra para a secagem das folhas.

Os tratamentos foram constituídos por dois métodos de secagem, o convencional pela estufa regulado a temperatura de $70^{\circ} \mathrm{C}$ e o FMO, conforme Lacerda et al. (2009), onde indicam submeter cada amostra a secagem a 3 ciclos de 5 minutos, 1 ciclo de 3 minutos, 1 ciclo de 2 minutos e 1 ciclo de 1 minuto (até atingir massa constante). Em cada intervalo ou ciclo regular, o material vegetal seco em forno de microondas foi desprendido do recipiente com uma espátula, a fim de evitar a fixação na sua superfície e também com intuito de se evitar possibilidade de combustão e determinado a massa do material. A secagem foi realizada para os dois métodos até o momento em que não houve alteração na massa seca do material vegetal, sendo que no método convencional feito realizado a medição da massa seca a cada 24 horas, e no método do FMO, no final de cada ciclo, logo após as amostras esfriarem. 
No interior do FMO, foi colocado dois bécker com $150 \mathrm{ml}$ de água a fim de umedecer o ambiente e evitar a queima das amostras e danos no aparelho ( UNDERSANDER et al., 1993) e a água será trocada a cada nova sequência para evitar que ferva e espirre na amostra alterando sua umidade e aumentando o tempo de secagem.

Após a obtenção da MS, as amostras foram moídas em moinho tipo Willey e acondicionadas em sacos de papel, para posterior determinação dos teores de macronutrientes, conforme indicações de Bataglia et al. (1983).

Foi utilizado como tratamento estatístico, um arranjo hierárquico $2 \times 3$ ( 2 métodos de secagem e 3 tipos de cultura), com 6 repetições, totalizando 36 amostras. O software utilizado para determinação dos resultados foi o SAS, ao nível de significância de 5\%.

\section{RESULTADOS E DISCUSSÃO}

Com relação a determinação do teor de matéria seca e aos teores de macronutrientes nas folhas das culturas de café, manga e amora, não apresentaram diferença significativa entre o método de secagem convencional e o FMO (Tabela 1).

Tabela 1. Teores foliares de nutrientes das culturas da amora, café, manga e a matéria seca (MS) das folhas em função dos métodos de secagem.

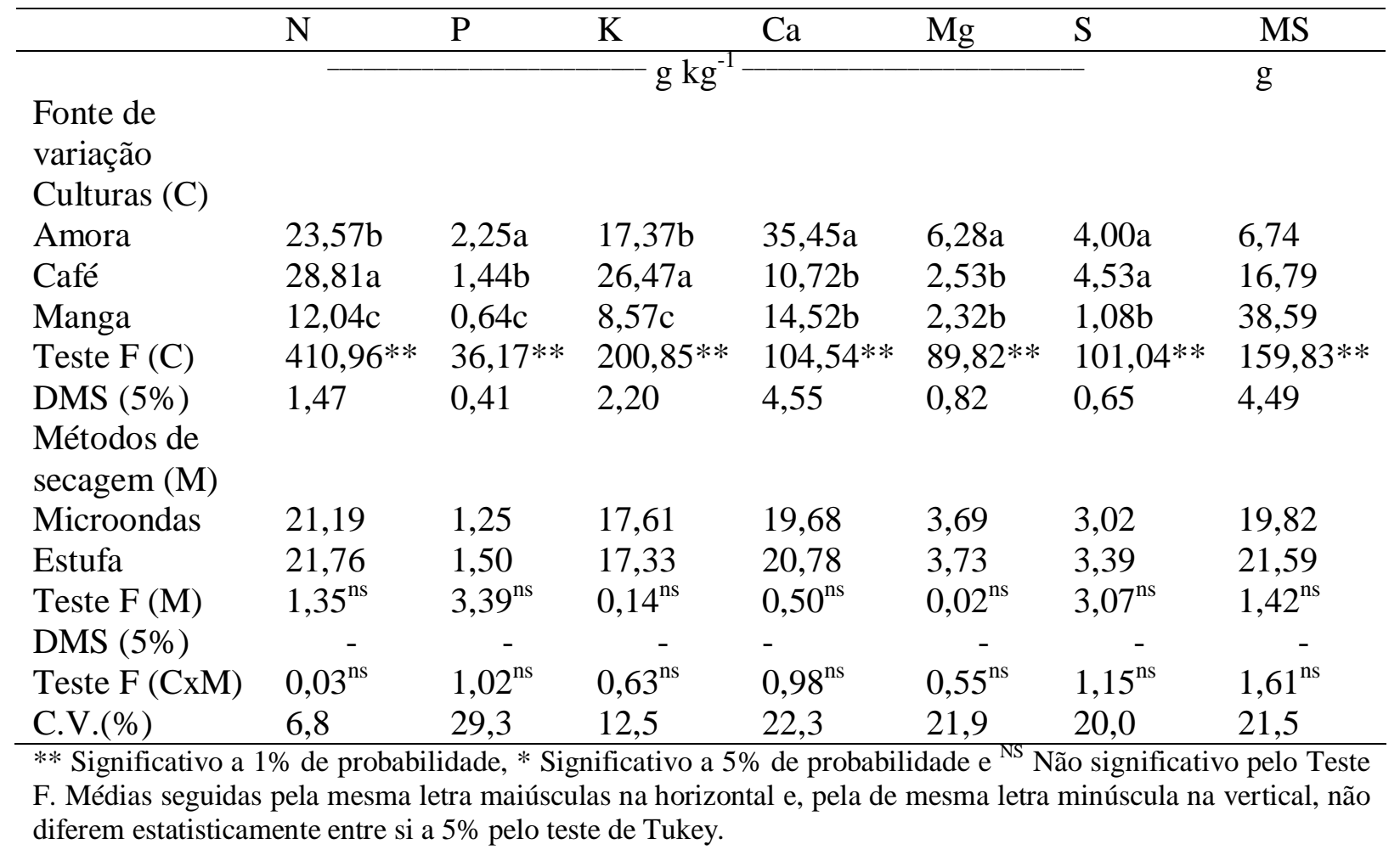

Nucleus, v.8, n.2, out.2011 
Observou-se que houve diferença dos teores de nutrientes entre as três culturas, o que era esperado devido tratar de espécies distintas (Tabela 1). Considerando os teores foliares de nutrientes médios ( 2 metodos de secagem) para a cultura do café nota-se que são considerados adequados segundo Raij et. al (1997) (N: 26-32 g/kg; P: 1,2-2,0 g/kg; K: 1,8-25 $\mathrm{g} / \mathrm{kg}$; Ca: 10-15 g/kg; Mg: 3,0-7,0 g/kg; S: 1,5-2,0 g/kg). Para os teores foliares de nutrientes médios para a cultura da manga (N: $12-14 \mathrm{~g} / \mathrm{kg}$; P: 0,8-1,6 g/kg; K: 5,0-10,0 g/kg; Ca: 20-35 $\mathrm{g} / \mathrm{kg} ; \mathrm{Mg}: 2,5-5,0 \mathrm{~g} / \mathrm{kg} ; \mathrm{S}: 0,8-1,8 \mathrm{~g} / \mathrm{kg}$ ). E para os teores foliares de nutrientes médios para a cultura da amora (N: 19,85-26,75 g/kg; P: 0,95-1,63 g/kg; K: 9,45-17,18 g/kg; Ca: 2,18-3,7 $\mathrm{g} / \mathrm{kg} ; \mathrm{Mg}: 2,18-3,7 \mathrm{~g} / \mathrm{kg} ; \mathrm{S}: 1,20-1,78 \mathrm{~g} / \mathrm{kg})$.

Ressalta-se pelos resultados obtidos que a ausência de diferenças entre os teores de macronutrientes nas três culturas avaliadas pelos dois métodos de secagem, concorda com Chang et al. (1994) sendo que a secagem de tecido vegetal pelo método FMO é adequado.

Pastorini et al. (2002) também observaram que quando comparados os dois métodos de secagem, em estufa com circulação de ar forçada e FMO, não houve diferenças significativas entre a matéria seca de plantas de milho e feijão e que também não houve alterações nos teores de aminoácidos, uma vez que o teor de aminoácidos é uma forma direta de mensuração do teor de nitrogênio presente na planta. Figueiredo et al. (2004) também comprovaram que o uso de microondas pode ser uma alternativa viável na determinação rápida dos teores de matéria seca dos diferentes estádios do ciclo vegetativo do capim elefante, podendo ser uma ferramenta eficiente para a determinação da umidade de forma rápida e precisa. Lacerda et al. (2009) comparando os dois tipos de secagem para obtenção de MS das forragens, demonstrou que o uso de FMO é uma alternativa mais rápida e com eficiência semelhante ao método de secagem em estufa com circulação de ar.

Chan et al. (2009), estudando a relação dos métodos de secagem na composição química de folhas de Alpinia zerumbet, E tlingera elatior, Curcuma longa e Aempferia galanga, observaram que não houve diferenças nas propriedades oxidantes dessas espécies das folhas secas por FMO ou por estufa com circulação de ar forçada.

Segundo Vinholis et al. (2007) o método avaliado de uso de microondas para determinação do conteúdo de água em amostras de plantas, assim como o presente estudo, permite agilizar o processo de controle da qualidade de recursos naturais e de insumos e otimizar atividades de alimentação e de irrigação em sistemas intensivos de produção agrícola, e que, quando em comparação com os procedimentos de rotina, gera impacto positivo do ponto de vista econômico, social e ambiental nos sistemas de controle da qualidade e de produção. 


\section{CONCLUSÃO}

O uso de FMO e da estufa com circulação de ar forçada na determinação da massa seca e nos teores de macronutrientes de folhas das culturas de café, manga e amora foram semelhantes, indicando que o uso do FMO é uma alternativa viável para uso em laboratórios de análise foliar.

\section{REFERÊNCIAS}

BATAGLIA, O.C.et al. Métodos de análise química de plantas. Campinas, Instituto Agronômico. Boletim Técnico, 78. 48p. 1983.

CHANG, H.et al. Effect of microwave treatment of the green color conservation and durability for bamboo. Quarterly Journal Forestry, London, v.27, n. 4, p.103-115, 1994.

FIGUEIREDO, M.P. et al. Determinação Do Teor De Matéria Seca Do Capim Elefante (Pennisetum purpureum Schum), Em Três Estádios De Maturidade Fisiologia, Pelo Forno de Microondas, Magistra, Cruz das Almas-BA, v.16, n.2, p.113-119, 2004.

GRASSI FILHO, H. Diagnose foliar: princípios e aplicações. In: PRADO, R.M.et al. Nutrição de plantas: diagnose foliar em grandes culturas. UNESP Jaboticabal, 2008, p.3560 .

HIGGINS, T.R.; SPOONER, A.E. Microwave drying of alfalfa compared to field-and- ovendrying: effects on forage quality. Animal Feed Science and Technology, Davis, v.16, n.1-2, p.1-6,1986.

HORSTEN, D. VON.; HARTNING, T.; VON HORTEN, D. Processing of medicinal plants using microwaves. Land Technik. Dusseldorf, v.54, n. 4, p.206-207, 1999.

LACERDA, M.J.R.; FREITAS, K.R.; SILVA, J.W. Determinação da matéria seca de forrageiras pelos métodos de microondas e convencional. Bioscience Journal, v.25, n.3, p.185-190, 2009.

MALAVOLTA, E. Manual de nutrição mineral de plantas. CERES, 2006. 631p

MARTINEZ, H. E. P.; CARVALHO, J. G.; SOUZA, R. B. Diagnose foliar. In: RIBEIRO, A. C.; GUIMARÃES, P. T. G.; ALVAREZ V., V. H. Recomendações para o uso de corretivos e fertilizantes em Minas Gerais - 5ª Aproximação. Viçosa: CFSMG., 1999. p.141-168.

MARUR, C.J.; SODEK, L. Microwave drying of plant material for biochemical analysis. Revista Brasileira de Fisiologia Vegetal, Viçosa, v.7, n.1, p.111-114, 1995.

NARASIMHALU, P.; KUNELIUS, H.T.; WINTER, K.A. Rapid determination of dry matter in grass silage of Lolium sp. using a microwave oven. Canadian Journal of Plant Science, Quebec, v.62, n.2, p.233-235, 1982. 
PASTORINI, L. H.; BACARIN, M. A.; ABREU, C. M. Secagem de material vegetal em forno de microondas para determinação de matéria seca e análises químicas. Ciência e Agrotecnologia, Lavras, v.26, n.6, p.1252-1258, 2002.

PRADO, R. M. Nutrição de Plantas. São Paulo: Editora UNESP, 2008. v.1. 407p.

QUAGGIO, J.A.; RAIJ, B. van.; PIZA JÚNIOR, C.T. Frutíferas. In: Recomendações de adubação e calagem para o Estado de São Paulo. RAIJ, B. Van et al. (Eds). 2. ed. rev. Campinas: IAC. 1997. p.121-125 (Boletim Técnico, 100).

QUAGGIO, J.A.; FURLANI A.M.C. (Eds). 2 Ed. rev. Campinas: IAC. 1997. p.233-236. (Boletim Técnico, 100)

RAIJ, B. van.; CANTARELLA, H. Outras culturas industriais. In: Recomendações de adubação e calagem para o Estado de São Paulo. RAIJ, B. van.; CANTARELLA, H.;

UNDERSANDER, D.R.; THIEX, N. Forage analyses procedures. Omaha: National Forage Testing Association. 1993. 153p.

VINHOLIS, M. M. B. Impacto Econômico, social e ambiental do uso do microondas doméstico para determinação de matéria seca e do teor de água em solos e plantas. Londrina, 22 a 25 de julho de 2007, Sociedade Brasileira de Economia, Administração e Sociologia Rural. 
